\title{
Simultaneous Contrast Restoration and Obstacles Detection: First Results
}

\author{
Nicolas Hautière, Jean-Philippe Tarel \\ DESE - LCPC \\ 58 Boulevard Lefebvre \\ 75732 Paris Cedex 15, France \\ \{hautiere, tarel\}@lcpc.fr
}

\author{
Didier Aubert \\ LIVIC - INRETS / LCPC \\ Bldg 824, 14 route de la Minière \\ 78000 Versailles, France \\ auberteinrets.fr
}

\begin{abstract}
Reliable obstacles detection under adverse weather conditions, especially foggy conditions, is a challenging task because the contrast is drastically reduced. Consequently, the classical approaches relying on pattern recognition techniques or points of interest matching are not so efficient anymore. In this paper, a novel approach is proposed which is able to simultaneously restore the contrast of the scene and to detect the presence of obstacles by stereovision once the atmosphere opacity is known. The different computation stages are detailed: fog density estimation, contrast enhancement, local distortions detection and obstacles detection, as well as their combination. The method is illustrated and partially assessed thanks to a video sequence under foggy weather. Finally, future research directions are indicated.
\end{abstract}

\section{INTRODUCTION}

Reliable obstacles detection is a fundamental task for the prevention of collisions, the inter-distance management [31]. Most reliable methods are based on multi-sensor fusion to ensure a high rate of detection and a very low rate of false detections [18]. In this area, RADAR, LIDAR and camera are among the most used sensors. One efficient multi-fusion strategy is to detect targets with one sensor, which are then confirmed by a second sensor [26]. However, this strategy has some limitations under degraded weather conditions, such as heavy rain or fog. Indeed, the operation range of optical sensors (LIDAR, camera) is reduced under these meteorological conditions. Consequently, the detections which would be provided by a RADAR would not be confirmed by another sensor.

A LIDAR can be used to determine bad weather conditions. Some strategies are proposed to enhance its performances under such conditions [29]. Cameras can also be used to detect low visibility conditions [11], [12], [14], [27]. A strategy has been proposed to enhance its performances for robust lane markings detection under bad weather conditions [10].

No strategy to mitigate the impact of weather conditions on obstacle detection has been proposed. The simpleminded approach consists in lowering the thresholds of low level image processings, such as gradients extraction. This is useless and even dangerous. Indeed, given that the contrast decay is exponential with respect to the depth of scene points, noisy features would be extracted close to the vehicle potentially causing some false detections and no new image features would be extracted further, which does not improve the detection rate.

In this paper, an original strategy is proposed based on stereovision which consists in simultaneously restoring the contrast and detecting the objects above the road surface.

This paper is organized as follows. In a first part, we recall why optical sensors are affected by adverse weather conditions. In a second part, the principle of our contrast restoration algorithm is introduced. In a third part, the "u-v disparity" stereovision framework to detect vertical objects above the road surface is recalled. In a fourth part, we present the principle of the simultaneous contrast restoration and detection algorithm. To illustrate the proposal, a video sequence under foggy weather is used in the paper. Finally, some research directions to validate and improve the proposed approach are indicated.

\section{Optical SEnsors AND Adverse WeAther CONDITIONS}

The operation range of exteroceptive sensors depends on the weather conditions. A study has been done in [16] to study the operation range of infrastructure based sensors with respect to weather conditions. The results can be extrapolated for in-vehicle sensors. Thus, according to the curves plotted in Fig. 1 and partially extracted from [2], the output signal of optical sensors running in the visible or near infrared light range is degraded by adverse weather conditions. Consequently, signal processings techniques relying on optical sensors to detect obstacles or the lane markings are less efficient under adverse weather conditions.

Thus, to be able to detect that the output signal of exteroceptive sensors is degraded by environmental conditions while relying only on the signal itself is a critical step. Indeed, it enables to automatically adapt the operation range of the sensors and the associated signal processing according to the encountered environmental conditions. Then, if this objective is reached, it will ensure a high reliability of future advanced driving assistances and allow their massive deployment.

\section{Contrast And Meteorological Visibility}

\section{A. Vision and the Atmosphere}

Koschmieder's law is a basic equation in daytime visual range theory relating the apparent luminance of a distant 


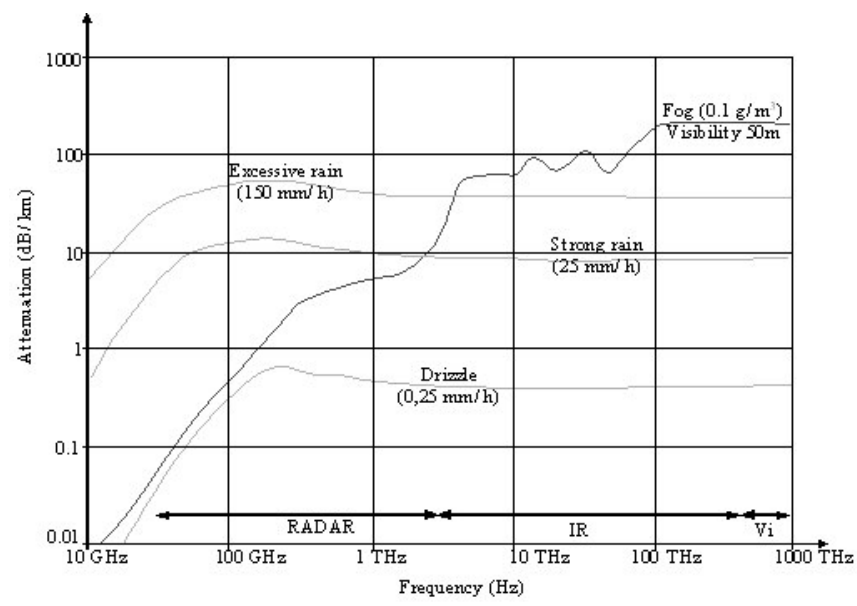

Fig. 1. Attenuation of electromagnetic signals with respect to the meteorological conditions [2]: drizzle, rain, heavy rain, excessive rain, fog. The three areas of the electromagnetic spectrum which are typically considered in in-vehicle sensors are indicated on the abscissa axis.

black object of intrinsic luminance $L_{0}$, the apparent luminance of the background sky above the horizon $L_{f}$, the extinction coefficient $k$ of the atmosphere and the distance of observation $d$ [22]. It is also called airlight formula [24]:

$$
L=L_{0} e^{-k d}+L_{f}\left[1-e^{-k d}\right]
$$

According to the International Commission on Illumination [1], for a contrast visibility threshold of $5 \%$, the meteorological visibility $V_{m e t}$ during the day can be related approximately to extinction coefficient using Koschmieder's relation (1):

$$
V_{m e t}=\frac{3}{k}
$$

The meteorological visibility distance is thus a standard dimension that characterizes the opacity of the atmosphere.

\section{B. Recovering the Atmospheric Extinction Coefficient}

For automotive applications, a camera or a LIDAR can be used to recover the value of the atmospheric extinction coefficient $k$.

\section{1) LIDAR:}

This laser based sensor can be used for estimating fog density by measuring the signal backscattered by fog droplets [4]. The power per pulse from range $r$ received by a LIDAR is given by the simplified LIDAR equation [6]:

$$
P_{R}(r)=\frac{P_{T} c \tau \beta A_{e}}{8 \pi r^{2}} \exp \left[-2 \int_{0}^{r} k(x) d x\right]
$$

where $P_{T}$ denotes the peak transmitted power per pulse, $c$ the speed of light, $\tau$ the pulse duration, $\beta$ the backscatter cross section per unit volume, $A_{e}$ the effective receiver aperture and $k(x)$ the atmospheric extinction coefficient.

Under strictly homogeneous conditions, $\beta$ and $k$ are not dependant of range. Hence, (3) becomes:

$$
P_{R}(r)=\frac{A^{”}}{r^{2}} \exp [-2 k r]
$$

where $A$ " denotes a constant that depends on device characteristics. The slope of the decaying waveform when differentiating (3) with respect to range becomes:

$$
\frac{d P_{R}(r)}{d r}=P_{R}(r)\left[-2 k-\frac{2}{r}\right]
$$

This differential equation is the basic principle on which the LIDAR measurement of the extinction coefficient in a homogeneous scattering medium is done.

For automotive applications, the LIDAR seems to be the best suited active sensor for estimating the meteorological visibility, since it does not need any receiver, contrary to infrastructure based visibilitymeters, and can be used for other safety applications, e.g. obstacles detection [26] or lane recognition [25]. Consequently, it has been used for adjusting the power of headlights [3], [28] or for adjusting the headway in Automatic Cruise Control (ACC) [5] according to the prevailing meteorological conditions. However, it has been shown that the dynamic adaptation of the emitting power of a LIDAR with respect to visibility conditions is not always perfect [7].

\section{2) Camera:}

In [14], [19], a method aiming at estimating the extinction coefficient of fog $k$ is presented. By replacing $d$ in (1) by the corresponding image line assuming a flat world and by taking twice the derivative of (1) with respect to the image line, yields a simple expression giving the parameter $k$ according to the positions of the inflection point $v_{i}$ and the horizon line $v_{h}$ :

$$
V_{m e t}=\frac{3 \lambda}{2\left[v_{i}-v_{h}\right]}
$$

where $\lambda=\frac{h \alpha}{\cos ^{2} \theta}$ depends on the camera parameters. $h$ denotes the sensor mounting height, $\alpha$ the ratio between the focal length of the camera and the pixel size assuming square pixels, and $\theta$ the pitch angle of the camera (see Fig. 3).

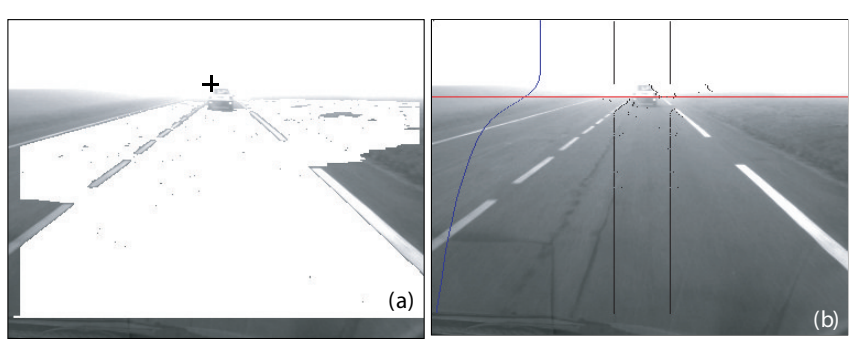

Fig. 2. Example of estimation of the meteorological visibility distance using the method described in [14], [19]: (a) partial detection of road and sky areas ; furthermore, the location of the vanishing point location (black cross) can be estimated in this way. (b) left curve: instantiation of (6) ; black segments: measurement bandwidth ; horizontal line: image line representative of the meteorological visibility distance. 
An example of automatic fog detection and estimation of the meteorological visibility distance is given in Fig. 2. Fig. 2(a) depicts the result of a region growing technique which detects some parts of the road and the sky. Thanks to the previous step, the instantiation of (1) is possible and finally the value of $V_{m e t}$ is automatically recovered by using (6). The image line representative of the meteorological visibility distance is plotted horizontally on Fig. 2(b).

\section{Enhancing the Contrast}

Once atmosphere opacity is known thanks to the computation of $k$, it is possible to restore the contrast of the images grabbed by the camera. The proposed approach consists in inverting the airlight model (1):

$$
L_{0}=L e^{k d}+L_{f}\left[1-e^{k d}\right]
$$

The missing parameters are now $L_{f}$ and a distance distribution in the scene. If $k$ is estimated thanks to the method described in [14], $L_{f}$ can be directly estimated. Otherwise, one can search the sky region directly [8], [21]. Moreover, an attempt to extract $L_{f}$ was proposed in [30].

Using onboard cameras, the distances distribution in the road scene is unknown a priori which is a problem. We explain in the next section how to infer a suited distances distribution which will help to understand the scene structure.

\section{Inferring a Suited Distance Distribution}

In the road context, the distances distribution in the scene can be roughly divided in two parts. A first part models the road surface which can be approximated by a plane. A second part models the objects above the road surface. According to [23], the depth of a scene point can be modeled as a function of the euclidian distance in the image plane between the corresponding pixel and the vanishing point $\left(u_{h}, v_{h}\right)$. Consequently, the depth $d$ of a pixel with $(u, v)$ coordinates can be represented as:

$$
d=\min \left[\frac{\lambda}{v-v_{h}}, \frac{\kappa}{\sqrt{\left(u-u_{h}\right)^{2}+\left(v-v_{h}\right)^{2}}}\right]
$$

where $\kappa>\lambda$ models the relative importance of the flat world against the vertical world.

Thus, by adjusting the value of $\kappa$, it will be possible in section $\mathrm{V}$ to simultaneously restore and detect vertical objects in a poor contrasted scene. Before presenting this algorithm, let us recall our stereovision framework aiming at detecting the objects above the road surface and a recent contribution to precisely fit bounding boxes around the objects [13].

\section{Stereovision Based RoAd Scene AnAlysis}

Thanks to the "u-v disparity" stereovision technique, it is possible to compute the longitudinal profile of the road, then to detect vertical planes and finally to set bounding boxes around the vertical objects above the road surface.

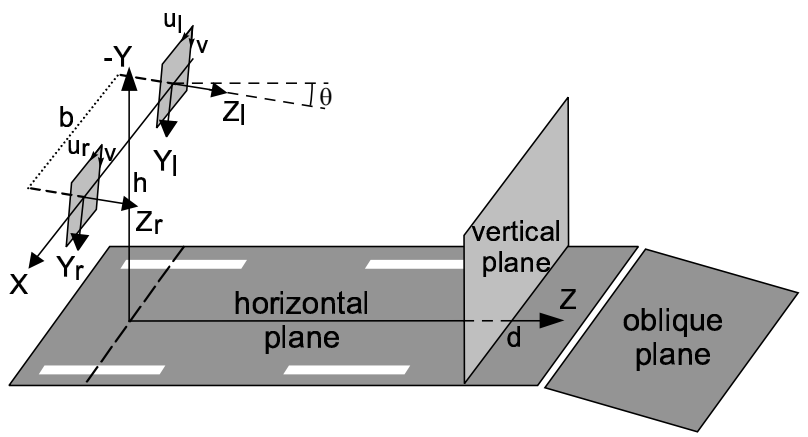

Fig. 3. Domain of validity of the study and coordinate systems used.

\section{A. The Image of a Plane in the "v-disparity" Image}

The stereovision algorithm uses the "v-disparity" transform, in which the detection of straight lines is equivalent to the detection of planes in the scene. In this aim, the $v$ coordinate of a pixel is represented towards the disparity $\Delta$ (performing accumulation from the disparity map along scanning lines) and detect straight lines and curves in this "v-disparity" image (denoted by $I_{v_{\Delta}}$ ) [17].

This algorithm assumes that the road scene is composed of set of planes: obstacles are modeled as vertical planes, whereas the road is supposed to be an horizontal plane (when it is planar), or a set of oblique planes (when it is not planar), as shown in Fig. 3.

According to the modeling of the stereo sensor given on Fig. 3, the plane of equation $Z=d$, corresponding to a vertical object, is projected as the straight line (9) in $I_{v_{\Delta}}$ :

$$
\Delta=\frac{b}{d}\left[v-v_{0}\right] \sin \theta+\frac{b}{d} \alpha \cos \theta
$$

The plane of equation $Y=0$, corresponding to the road surface, is projected as the straight line (10) in $I_{v_{\Delta}}$ :

$$
\Delta=\frac{b}{h}\left[v-v_{0}\right] \cos (\theta)+\frac{b}{h} \alpha \sin \theta
$$

The different parameters of the sensor are the same as in section III-B.2 except that $b$ is the distance between the cameras (i.e. the stereoscopic base) and $\left(u_{0}, v_{0}\right)$ denotes the position of the optical center in the image coordinate system. Mathematical details can be found in [17].

\section{B. "v-disparity" Image Construction and 3-D Surface Ex- traction}

The algorithm performs a robust extraction of these planes from which it deduces many useful information about the road and the obstacles located on its surface. From two stereo images, a disparity map $I_{\Delta}$ is computed (Zero Normalized Cross Correlation -ZNCC- criteria is used to this purpose along edges). Then an accumulative projection of this disparity map is performed to build the "v-disparity" image $I_{v \Delta}$. For the image line $i$, the abscissa $u_{M}$ of a point $M$ in $I_{v \Delta}$ corresponds to the disparity $\Delta_{M}$ and its grey level $i_{M}$ to the 
number of points with the same disparity $\Delta_{M}$ on the line $i: i_{M}=\sum_{P \in I_{\Delta}} \delta_{v_{P}, i} \delta_{\Delta_{P}, \Delta_{M}}$ where $\delta_{i, j}$ denotes the Kronecker delta.

>From this "v-disparity" image, a robust extraction of straight lines is performed through a Hough transform. This extraction of straight lines is equivalent to the extraction of the planes of interest taken into account in the modeling of the road scene (cf. Fig. 3).

\section{C. "u-disparity" Image Computation and Objects Segmenta- tion}

Once vertical planes are extracted, a bounding box has to be positioned around the different vertical objects on the road surface. To compute their lateral position, it is proposed in [17]to perform an accumulative projection of this disparity map along the horizontal axis, in order to build a "u-disparity" image $I_{u \Delta}$. Unfortunately, left and right sides of obstacles are often disconnected. This is due to the fact the technique relies only on horizontal gradients to match the stereo pairs. So the disparity can not be computed on horizontal edges. In this way, objets composed of both vertical and horizontal gradients can not be correctly detected, like the backside of a vehicle for example.

To solve the problem, we propose to propagate disparity information on horizontal edges using the quasi-dense matching algorithm developed in [20]. Its principle is to perform a region growing in the disparity space, guided not by a criterion of homogeneity but by a score of correlation, in order to progressively densify the disparity map into textured areas.

Thus, the seed matches of the quasi-dense matching algorithm are the matches used to compute the "v-disparity" image. Then, the initial seeds are propagated like in [20], except that for each match candidate we check if it belongs to one of the planes of the "v-disparity" image. If it is the case, the match candidate is added to the current set of seeds and to the current set of accepted matches, which is under construction. Otherwise, the match candidate is removed from the current set of seeds.

Resulting of the proposed method, the disparity map is quasi-dense, especially on the vertical objects (Fig. 5(c)). We can thus compute a quasi-dense and reliable "u-disparity" image and set an accurate bounding box around objects (Fig. 5(d)). Thus, using this approach, the risk that the same object is split into different bounding boxes is reduced. Details of the method can be found in [13].

\section{Simultaneous Contrast Restoration And OBSTACle DeteCtion}

\section{A. Principle}

Initialized with a small initial value of $\kappa$ in (8), e.g. $\kappa=$ $1.1 \lambda$, the principle of the simultaneous contrast restoration and obstacle detection algorithm is to progressively increase the value of $\kappa$ and to detect the distorted areas. As soon as vertical objects are encountered, a local contrast distortion can be noticed. In this case, the vertical object causing the distortion is detected by stereovision. The increase of

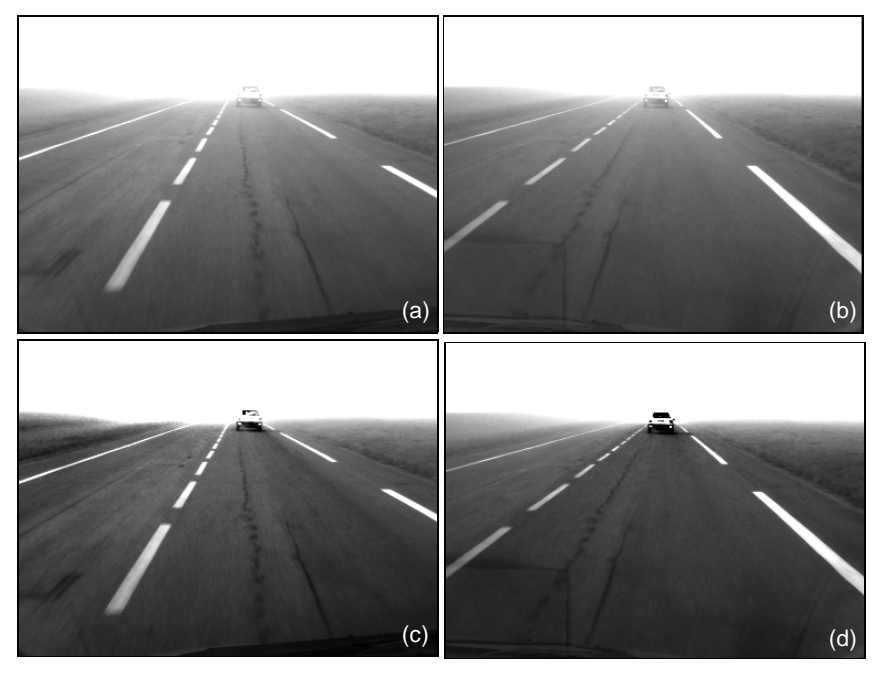

Fig. 4. Illustration of the contrast enhancement process. (a)(b) Original stereo pair. (c)(d) "Half-restored" images. The contrast restoration process created some visual distorsions in right image on the vehicle (Fig. 5a). The contrast restoration parameters are then used to restore the contrast of the left image.

$\kappa$ can then be restarted until the desired final value, e.g. $\kappa=10 \lambda$, is reached. Let us describe more precisely the different computation stages of the proposed algorithm.

\section{B. Contrast Restoration}

The contrast restoration stage consists in inverting Koschmieder's law (7). The distance distribution in the scene is the one of (8) with the given value of $\kappa$. For the pixels belonging to the road surface, the contrast is correctly restored. For the pixels corresponding to vertical objects, as the value of $\kappa$ increases, the contrast is not correctly restored. On the contrary, because their distances is overestimated (they are assumed to belong to the road surface), their contrast is also overestimated. Consequently, their intensities become null even negative and creates visual distortions.

Such visual distortions can be noticed on Figs. 4(c)(d) on the pixels corresponding to the rear part of the followed vehicle. The white pixels remain white and the grey pixels become black after contrast enhancement. They are precisely detected thanks to the method described in the next paragraph.

\section{Local Distortions Detection}

The overestimation of scene points distance creates some black and white visual distortions in the image. To momentarily stop the contrast restoration process and to detect the corresponding objects, these distortions must be detected. In this aim, we propose to detect a decrease of local normalized correlation between the original image and the restored image. Indeed, if the contrast is correctly restored, the normalized correlation should remain constant. Indeed, thanks to the normalization, the proposed image quality attribute is not sensitive to contrast change. However, when the contrast is distorted, the normalized correlation will 


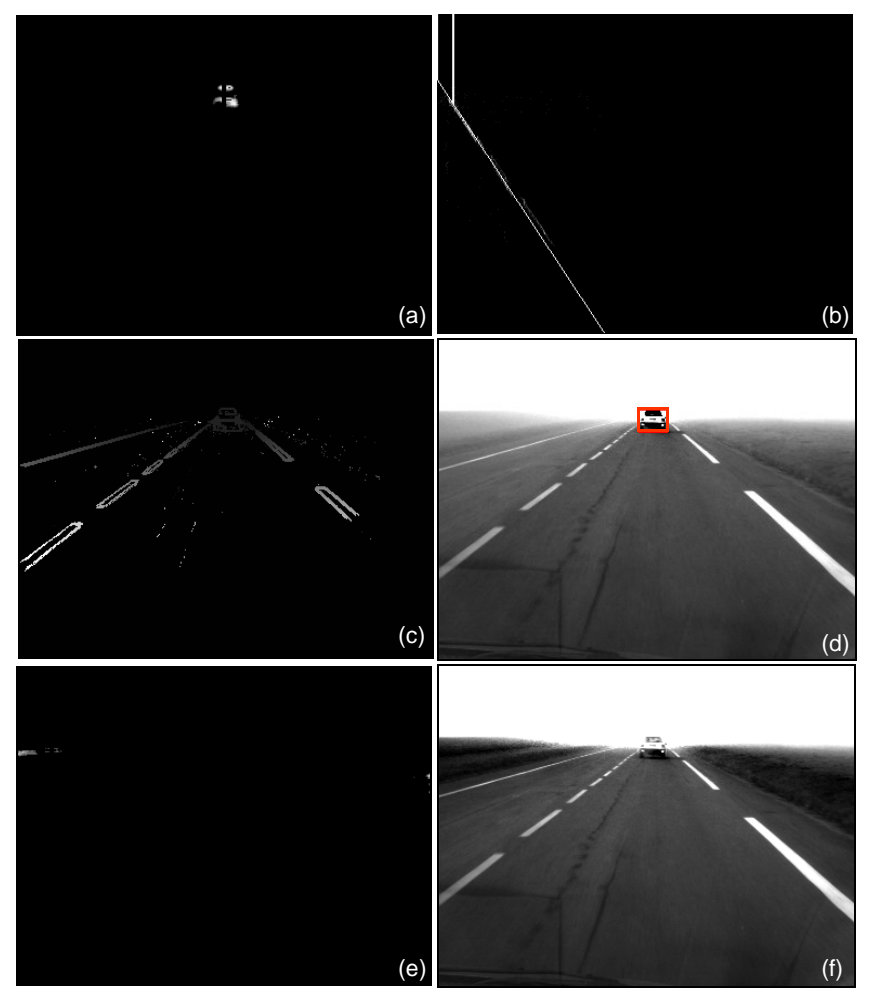

Fig. 5. (a) Distorted pixels after the initial contrast restoration process ; (b) "v-disparity" image computed using the stereo pair given on the Figs. 4(c)(d) ; (c) quasi-dense disparity map obtained using the method given in [13] ; (d) bounding box around the detected vehicle computed thanks to "u-disparity" ; (e) distorted pixels after fi nal contrast restoration ; (f) fi nal image.

decrease. Once local distortion is detected, the corresponding object bounding box can be extracted by stereovision as explained in next section.

Two examples of detection of local distorted pixels are given and Figs. 5(a)(e). Fig. 5(a) corresponds to the distorted pixels of the initial restoration process. Fig. 5(e) corresponds to the distorted pixels of the final restoration process.

\section{Obstacle Detection}

To detect the object which corresponds to a local distortion, the "u-v disparity" stereovision approach is used which was presented in section IV. Thus, assuming that the right image of the stereo pair is used to restore the contrast, the restoration parameters, especially the $\kappa$ value, are used to restore the contrast of the left image of the stereo pair. The detection of the object is then possible and is facilitated thanks to the contrast distortion for two reasons. Firstly, the contrast is enhanced so that the object is easier to segment. Secondly, the stereovision algorithm must only confirm that the local distorted pixels correspond to an object of interest.

Thus, a "v-disparity" image is computed and the different vertical planes corresponding to obstacles are detected (Fig. 5b). A quasi-dense disparity map is computed (Fig. 5c). Finally, a bounding box around the vertical objects is fitted (Fig. 5d). Their true distances can be estimated. Consequently, the contrast can be correctly restored on them.
Thereafter, the contrast restoration loop can then restart outside the area where the obstacle has been detected. When the final value of $\kappa$ is reached, the contrast of the image is restored and the vertical objects have been detected, see Fig. 5(d)(f).

\section{E. Contrast Restoration Assessment}

To assess the proposed contrast restoration algorithm, we propose to compute the local contrasts above 5\% in the image before and after the contrast restoration. The proposed method is based on Köhler's binarization technique [15] and is detailed in [12]. Results are given on Fig. 6 for the right image of our test stereo pair. The increasing of the mobilized visibility distance [12], i.e. the distance to the most distant picture element belonging to the road surface having a contrast above $5 \%$, is clearly noticeable.

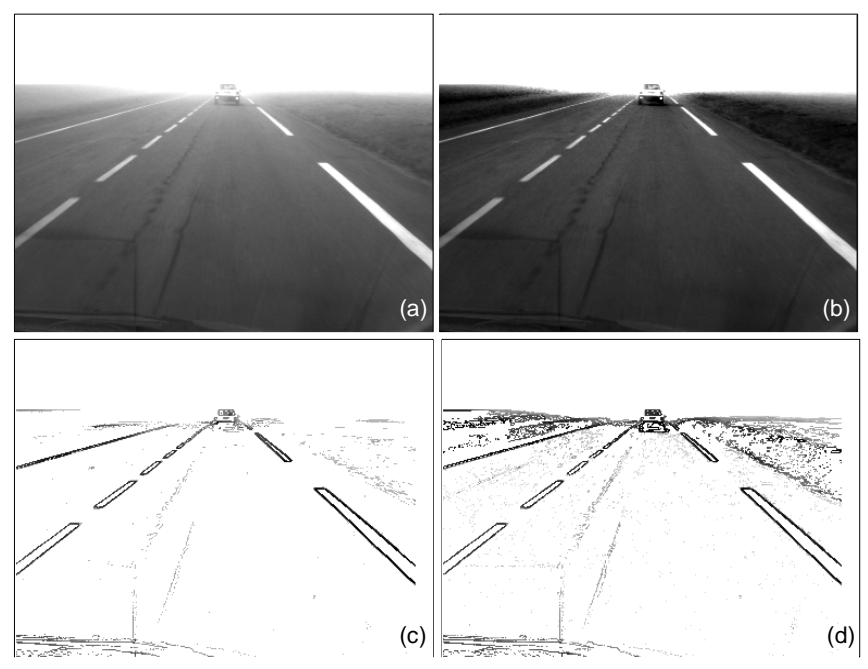

Fig. 6. (a) Original right image of the test stereo pair ; (b) image with restored contrast ; (c) local contrasts above 5\% computed on the original image ; (d) local contrasts above $5 \%$ on the image with restored contrast. The grey level of the pixels is proportional to the contrast value.

The proposed simultaneous contrast restoration and obstacle detection algorithm is summarized on Fig. 7.

\section{Discussion AND PERspectives}

In this paper, the first results of a novel approach to restore the contrast of weather degraded images and to detect obstacles by means of stereovision have been presented. This approach is novel because the contrast restoration step and the obstacles detection are done simultaneously. The proposed algorithm combines three technical components: a fog detection component, a contrast restoration component with its associated image quality attribute and an obstacles detection component. For the moment, the computational cost of the method is quite important (approximately 3-5 seconds for the test image) but no code optimization has been done. Then, an experimental validation of the different components must be carried out. Different scenarios with various meteorological conditions must be grabbed. In this 


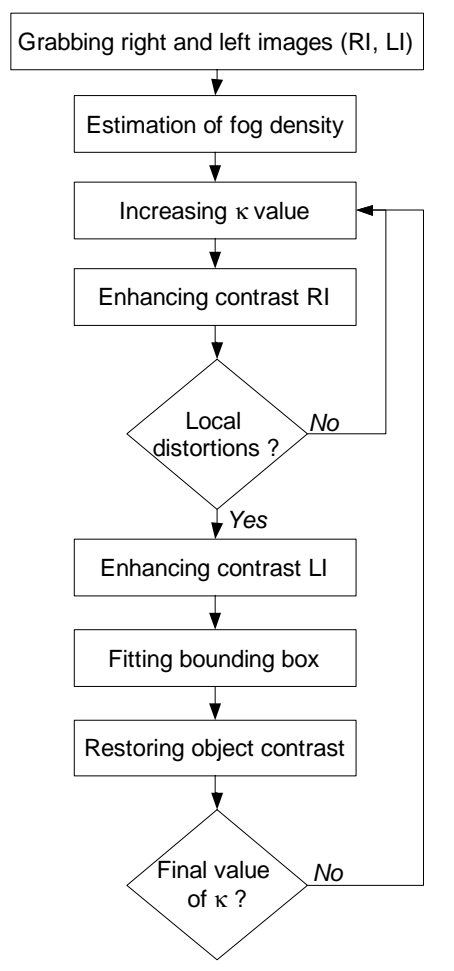

Fig. 7. Overview of the proposed simultaneous contrast restoration and obstacle detection algorithm (LI Left Image, RI Right Image).

context, the use of a virtual sensors simulation plateform may be useful [9].

Driving assistances which are able to deal with adverse conditions are not yet ready for deployment. Thus, the robustness of driving assistances is one of the priorities of the 7th Framework Programme of the European Commission which is just starting. In this context, to detect the unfavorable weather and lighting conditions by only using the output signal of the sensors themselves is one of the keys of the success. The approach in this paper presents such a contribution.

\section{REFERENCES}

[1] International Lighting Vocabulary. Number 17.4. Commission Internationale de l'Éclairage, 1987.

[2] P. Bhartia and I.J. Bahl. Millemeter Wave Engineering and Applications. John Wiley and Sons, 1984.

[3] C. Boehlau. Optical sensors for AFS - supplement and alternative to GPS. In Proc. Progress in Automotive Lighting Symposium, 2001.

[4] R. Brown. A new lidar for meteorological application. Journal of Applied Meteorology, 12(4):698-708, 1973.

[5] P. Carrea and A. Saroldi. Integration between anticollision and AICC functions: The ALERT project. In Proc. Intelligent Vehicles Symposium, 1993.

[6] R. Collis. Lidar. Applied Optics, 9(8):1782-1788, 1970.

[7] M. Colomb et al. The main results of a european research project: "improvement of transport safety by control of fog production in a chamber" ("FOG"). In International Conference on Fog, Fog Collection and Dew, October 2004.

[8] M. Fang, M.-Y. Chiu, C.-C. Liang, and A. Singh. Skyline for videobased virtual rail for vehicle navigation. In IEEE Intelligent Vehicles Symposium, pages 207-212, 1993.
[9] D. Gruyer, C. Royère, J.-M. Blosseville, G. Michel, and N. Du Lac. SiVIC and RTMaps, interconnected platforms for the conception and the evaluation of driving assistance systems. In ITS World Congress, London, UK, 2006.

[10] N. Hautière and D. Aubert. Contrast restoration of foggy images through use of an onboard camera. In Proc. IEEE Conference on Intelligent Transportation Systems, pages 1090-1095, September 2005.

[11] N. Hautière, R. Labayrade, and D. Aubert. Detection of visibility conditions through use of onboard cameras. In Proc. IEEE Intelligent Vehicles Symposium, 2005.

[12] N. Hautière, R. Labayrade, and D. Aubert. Real-Time Disparity Contrast Combination for Onboard Estimation of the Visibility Distance. IEEE Transactions on Intelligent Transportation Systems, 7(2):201212, June 2006

[13] N. Hautière, R. Labayrade, M. Perrollaz, and D. Aubert. Road scene analysis by stereovision: a robust and quasi-dense approach. In IEEE International Conference on Automation, Robotics, Control and Vision, 2006.

[14] N. Hautière, J.-P. Tarel, J. Lavenant, and D. Aubert. Automatic Fog Detection and Estimation of Visibility Distance through use of an Onboard Camera. Machine Vision and Applications Journal, 17(1):820, April 2006.

[15] R. Köhler. A segmentation system based on thresholding. Graphical Models and Image Processing, 15:319-338, 1981.

[16] R. Kurata, H. Watanabe, M. Tohno, T. Ishii, and H. Oouchi. Evaluation of the detection characteristics of road sensors under poor-visibility conditions. In Proc. IEEE Intelligent Vehicles Symposium, 2004.

[17] R. Labayrade, D. Aubert, and J.-P Tarel. Real time obstacle detection in stereovision on non fat road geometry through v-disparity representation. In Proc. IEEE Intelligent Vehicles Symposium, 2002.

[18] R. Labayrade, C. Royère, D. Gruyer, and D. Aubert. Cooperative fusion for multi-obstacles detection with use of stereovision and laser scanner. Autonomous Robots, 19(2):117-140, 2005.

[19] J. Lavenant, J.-P. Tarel, and D. Aubert. Procédé de détermination de la distance de visibilité et procédé de détermination de la présence d'un brouillard. Brevet français 0201822 soumis par LCPC / INRETS, Février 2002.

[20] M. Lhuillier. Match propagation for image-based modeling and rendering. IEEE Transactions on Pattern Analysis and Machine Intelligence, 24(8):1140-1146, 2002.

[21] W.-N. Lie, T.C.-I. Lin, T.-C. Lin, and K.-S. Hung. A robust dynamic programming algorithm to extract skyline in images for navigation. Pattern Recognition Letters, 26(2):221-230, 2005.

[22] W.E.K. Middleton. Vision through the atmosphere. University of Toronto Press, 1952.

[23] S. G. Narashiman and S. K. Nayar. Interactive deweathering of an image using physical model. In Proc. IEEE Workshop on Color and Photometric Methods in Computer Vision, 2003.

[24] S. G. Narasimhan and S. K. Nayar. Vision and the atmosphere. International Journal of Computer Vision, 48(3):233-254, 2002.

[25] T. Ogawa and K. Takagi. Lane recognition using on-vehicle lidar. In IEEE Intelligent Vehicles Symposium, 2006.

[26] M. Perrollaz, R. Labayrade, C. Royère, N. Hautière, and D. Aubert. Long range obstacle detection using laser scanner and stereovision. In IEEE Intelligent Vehicles Symposium (IV'06), Tokyo, Japan, pages 182-187, June 13-15 2006.

[27] D. Pomerleau. Visibility estimation from a moving vehicle using the RALPH vision system. In Proc. IEEE Conference on Intelligent Transportation Systems, November 1997.

[28] F. Rosenstein. Intelligent rear light - constant perceptibility of light signals under all weather conditions. In Proc. International Symposium on Automotive Lighting, pages 403-414, September 2005.

[29] R. Schulz and K. Fuerstenberg. Laserscanner for multiple applications in passenger cars and trucks. In International Conference on Advanced Microsystems for Automotive Applications, Berlin, Germany, 2006.

[30] S. Shwartz, E. Namer, and Y. Y. Schechner. Blind haze separation. In IEEE International Conference on Computer Vision and Pattern Recognition, volume 2, pages 1984-1991, 2006.

[31] S. Zehang, G. Bebis, and R. Miller. On-road vehicle detection: a review. IEEE Transactions on Pattern Analysis and Machine Intelligence, 28(5):694- 711, 2006. 